\title{
STATES AND REPRESENTATIONS
}

\author{
BY \\ RICHARD V. KADISON( ${ }^{(1)}$
}

1. Introduction. In [19], Murray and von Neumann made use of their trace function on a factor of type $\mathrm{II}_{1}$ to induce an inner product on the factor and, by left multiplication, a representation of the factor (with simple multiplicity). Gelfand and Neumark [5] employed a similar process in more general circumstances to construct representations of certain Banach algebras and prove their well-known characterization of $C^{*}$-algebras. Segal [24] recognized the importance of this construction of representations for the study of infinite-dimensional representations of locally compact groups and for the study of $C^{*}$-algebras themselves. Emphasizing the order structure, he constructed representations corresponding to positive linear functionals on a $C^{*}$-algebra. By means of such representations, spectral values could be made to correspond to eigenvectors. The key to many of the results of [24] was Segal's extremal characterization of those functionals which give rise to irreducible representations.

The program of this paper is to describe, in terms of the $C^{*}$-algebra and its space of positive linear functionals (states) the type invariants of the representing operator algebra arising from the given $C^{*}$-algebra and the representation induced by a given state of it. In particular, we characterize those states which give rise to a representing algebra whose weak closure is a factor, is of type I, of type II, and of type III (Theorem A). We shall sketch a technique for doing the dimension theories of the weak closure of the representing operator algebra due to a state and of its commutant in terms of the state space. (The "coupling" can be described in these terms, from this.)

For the sake of clarity, we have carried this program out for factor representations (the factor, or local, case). In the last section, we supply the definitions (Definitions 4.2 and 4.3) necessary to make the extension of these local results to the global case (arbitrary states) a routine matter. (The key to these results, Theorem C, applies to the global case.) This same section contains a characterization (Theorem $\mathrm{H}$ ) of those (norm) separable $C^{*}$-algebras which admit maximal cyclic representations (equivalent to no proper subrepresentations of cyclic representations). These are the separable type I (equivalently, GCR $[6 ; 14]$ ) algebras with countable structure space.

A standard translation makes these results applicable to unitary representations of locally compact groups.

2. Notation and preliminaries. Our $C^{*}$-algebras are complex Banach alge-

Received by the editors June 29, 1961.

(1) Alfred P. Sloan Research Fellow. 
bras with an operation $A \rightarrow A^{*}$ satisfying $(a A+B)^{*}=\bar{a} A^{*}+B^{*},(A B)^{*}$ $=B^{*} A^{*},\left(A^{*}\right)^{*}=A$, and $\left\|A A^{*}\right\|=\|A\| \cdot\left\|A^{*}\right\|$, and containing a unit element $I$. (This last is not an essential requirement.) A representation (sometimes called, ${ }^{*}$ representation) of such an algebra is a homomorphism $\phi$ whose image lies in the algebra of bounded operators on some Hilbert space, satisfying $\phi\left(A^{*}\right)=\phi(A)^{*}$.

A positive element $A$ in a $C^{*}$-algebra is one which is self-adjoint $\left(A=A^{*}\right)$ and whose spectrum consists of non-negative real numbers. A state $\rho$ of a $C^{*}$-algebra $\mathfrak{A}$ is a linear functional on $\mathfrak{A}$ such that $\rho(A) \geqq 0$ if $A \geqq 0$. (The requirement $\rho(I)=1$ is often added; but we shall not do so.) Each state $\rho$ of a $C^{*}$-algebra $\mathfrak{A}$ "induces" a representation of $\mathfrak{A}$ as follows $[5 ; 24]$. The mapping $A, B \rightarrow \rho\left(B^{*} A\right)$ is a positive semidefinite inner product on $\mathfrak{A}$. From the Cauchy-Schwarz inequality, the set of null vectors $\mathfrak{K}_{\rho}$ is a left ideal in $\mathfrak{A}$. The vector space quotient of $\mathfrak{A}$ by $\mathfrak{K}_{\rho}$ is then a pre-Hilbert space in the induced inner product. The operators $\phi_{\rho}(A)^{\prime}$ defined on the quotient by $\phi_{\rho}(A)^{\prime}\left(B+\mathfrak{K}_{\rho}\right)$ $=A B+\mathcal{K}_{\rho}$ are bounded and have unique bounded extensions $\phi_{\rho}(A)$ to the completion $\mathfrak{H}_{\rho}$. The mapping $\phi_{\rho}$ is the desired representation of $\mathfrak{A}$. The vector $x_{\rho}\left(=I+\mathfrak{K}_{\rho}\right)$ is cyclic under $\phi_{\rho}(\mathfrak{A})$, i.e., its images under $\phi_{\rho}(\mathfrak{A})$ form a dense subset of $\mathfrak{H C}_{\rho}$.

If the $C^{*}$-algebra $\mathfrak{A}$ acts on the Hilbert space $\mathfrak{H C}$ (i.e., the abstract $C^{*}$ algebra has a faithful representation on $\mathfrak{F}$ ), each vector $x$ in $\mathfrak{H C}$ defines a state of $\mathfrak{A}$ by $A \rightarrow(A x, x)$. If $\mathfrak{A}$ happens to be all bounded operators on $\mathfrak{H}$, we denote this state by $\omega_{x}$, otherwise, by $\omega_{x} \mid \mathfrak{A}$. We refer to it as a vector state of $\mathfrak{A}$. In the construction above, $\rho=\omega_{x_{\rho}} \circ \phi_{\rho}$ (i.e., $\rho(A)=\left(\phi_{\rho}(A) x_{\rho}, x_{\rho}\right)$, for each $A$ in $\mathfrak{A}$ ).

We speak of a representation such that there is a cyclic vector for the image as a cyclic representation. If $\phi$ and $\psi$ are cyclic representations of the $C^{*}$-algebra $\mathfrak{A}$ with cyclic vectors $x$ and $y$, respectively, such that $\omega_{x} \circ \phi$ $=\omega_{y} \circ \psi$, then the mapping $U^{\prime}$ defined by $U^{\prime}(\phi(A) x)=\psi(A) y$ extends to a unitary transformation $U$ of the Hilbert space upon which $\phi(\mathfrak{A})$ acts on to the Hilbert space upon which $\psi(\mathfrak{A})$ acts; and $U \phi(A) U^{-1}=\psi(A)$, for each $A$ in $\mathfrak{A}$. In general, when such a $U$ exists, we say that $\phi$ and $\psi$ are unitarily equivalent representations and that $U$ effects or implements the equivalence.

If $\mathcal{F}$ is a family of operators on and $V$ a set of vectors in the Hilbert space $\mathcal{F}$, we denote by [FU] the closure of the set of vectors $\{T x: T$ in $\mathcal{F}, x$ in $\mathcal{U}\}$. We denote by this same symbol the orthogonal projection operator on that subspace. In fact, we adopt our usual convention of identifying a subspace and the orthogonal projection on it with regard to terminology and notation. If $\phi$ is a representation of the $C^{*}$-algebra $\mathfrak{A}$ as operators on $\mathfrak{H C}$ and $x$ is in $\mathfrak{H C}$, then $[\phi(\mathfrak{A}) x]$ is a projection commuting with $\phi(\mathfrak{A})$. We call the set of operators commuting with a family $\mathcal{F}$, the commutant of $\mathcal{F}$ and denote it by $\mathcal{F}^{\prime}$. With $E^{\prime}$ a projection in $\phi(\mathfrak{A})^{\prime}$, the mapping $A \rightarrow \phi(A) E^{\prime}$ is a representation of $\mathfrak{A}$. Such a representation is called a subrepresentation of $\phi$. If, in particular, $E^{\prime}=[\phi(\mathfrak{A}) x]$, then this subrepresentation is unitarily equivalent to that in- 
duced by the state $\omega_{x} \circ \phi$ of $\mathfrak{A}$ (from the preceding paragraph). We denote by $\mathfrak{F}^{-}$the strong operator closure of the family of operators $\mathfrak{F}$. With $\mathcal{F}$ a $*$-algebra, $\mathfrak{F}^{-}$coincides with the weak operator closure of $\mathcal{F}[1$, p. 43, Theorem 2$]$. We note that $\left[\mathcal{F V}^{-}\right]=\left[\mathfrak{F}^{-\mathcal{V}}\right]$, for general $\mathcal{F}$.

If $\rho$ is a state of the $C^{*}$-algebra $\mathfrak{A}$ such that $\tau \leqq \rho$, for another state $\tau$ of $\mathfrak{A}$ (i.e., $\rho-\tau$ is a state of $\mathfrak{A}$ ) implies that $\tau=a \rho$, for some scalar $a$; we say that $\rho$ is a pure state of $\mathfrak{A}$. In another form, with $\rho \neq 0, \rho$ is pure if and only if $\rho / \rho(I)$ is an extreme point of the (convex) set of states which take the value 1 at $I$. In [24, pp. 79-80], the pure states are identified as those which induce irreducible representations (i.e., representations $\phi$ such that $\phi(\mathfrak{A})^{\prime}$ consists of scalar multiples of $I$, or, equivalently [20], $\phi(\mathfrak{A})^{-}$is all bounded operators). If $\rho$ is a state of the $C^{*}$-algebra $\mathfrak{A}$ and $A$ an operator in $\mathfrak{A}$, we denote by $\rho_{A}$ the state of $\mathfrak{A}$ defined by $\rho_{A}(B)=\rho\left(A^{*} B A\right)$.

Definition 2.1. If $\rho$ is a state of the $C^{*}$-algebra $\mathfrak{A}$, we denote by $\tilde{\rho}$ the norm closure of the set $\left\{\rho_{A}: A\right.$ in $\left.\mathfrak{A}\right\}$ in the dual space of $\mathfrak{A} ;$ and by $\bar{\rho}$ the norm closure of the set $\{\tau: \tau \leqq a \rho, \tau$ a state of $\mathfrak{A}\}$. We write $\tau \lesssim \rho$ when $\tilde{\tau} \subseteq \tilde{\rho}$, and $\tau \sim \rho$ when $\tilde{\tau}=\tilde{\rho}$. When $\tilde{\tau} \cap \tilde{\rho}=\{0\}$, we say that $\tau$ and $\rho$ are disjoint.

REMARKS (2a). The relation $\sim$ is an equivalence relation on the states of $\mathfrak{A}$.

(2b). The relation $\lesssim$ is (more precisely, induces) a partial ordering of the set (of equivalence classes) of states of $\mathfrak{A}$.

(2c). We have $\tau \lesssim \rho$ if and only if $\tau \in \tilde{\rho}$. In fact, if $\tau \lesssim \rho$, then $\tau \in \tilde{\rho}$ since $\tau \in \tilde{\tau}$. If $\tau \in \tilde{\rho}$, then, for some sequence of operators $\left(A_{n}\right)$ in $\mathfrak{A},\left\|\rho_{A_{n}}-\tau\right\| \rightarrow 0$. But $\left\|\rho_{A A_{n}}-\tau_{A}\right\| \leqq\left\|\rho_{A_{n}}-\tau\right\| \cdot\|A\|^{2}$; so that $\tau_{A} \in \tilde{\rho}$, for each $A$ in $\mathfrak{A}$. Thus $\tilde{\tau} \subseteq \tilde{\rho}$.

(2d). We noted that $\phi_{\rho}$ is irreducible if and only if $\rho$ is pure. Now $\mathcal{K}_{\rho}$ is one (or zero)-dimensional if and only if $\rho / \rho(I)$ is a homomorphism. Thus each homomorphism of $\mathfrak{A}$ is a pure state.

We shall make use of some of Mackey's terminology [16], applied, however, to representations of $C^{*}$-algebras rather than groups.

DEFINITION 2.2. If $\phi$ is a representation of the $C^{*}$-algebra $\mathfrak{A}$ as bounded operators on the Hilbert space $\mathfrak{H}$, we say that $\phi$ is a factor representation when $\phi(\mathfrak{A})^{-}$ has center consisting of scalar multiples of its unit element (i.e., is a factor in the sense of [18]). Two representations of $\mathfrak{A}$ are said to be disjoint when no subrepresentation of one is unitarily equivalent to a nonzero subrepresentation of the other.

We classify states according to the type invariants of the representing algebras corresponding to them.

DEFINITION 2.3. If $\rho$ is a state of the $C^{*}$-algebra $\mathfrak{A}$, we say that $\rho$ is primary if $\phi_{\rho}$ is a factor representation. If $\phi_{\rho}(\mathfrak{H})-$ is of type I, II, or III, we say that $\rho$ is of type I, II, or III, respectively, and that $\rho$ is finite or infinite as $\phi_{\rho}(\mathfrak{A})-$ is.

3. Principal results. Theorem A. If $\rho$ is a state of the $C^{*}$-algebra $\mathfrak{A}$ then $\rho$ is primary if and only if $\lesssim$ totally orders $\tilde{\rho}$. If $\rho / \rho(I)$ is not a homomorphism; $\rho$ is pure if and only if $\rho$ is minimal but not maximal in the set of primary states with 
respect to $\precsim$. If $\rho / \rho(I)$ is primary, it is of type I if and only if $\rho \precsim \tau$ for some pure state $\tau$ of $\mathfrak{A}$; it is of type III if and only if it is minimal and maximal in the set of primary states and not a homomorphism; it is of type II otherwise (equivalently, if $\tau \precsim \rho$ implies $\tau$ is not minimal).

Theorem B. A primary state $\rho$ of the $C^{*}$-algebra $\mathfrak{A}$ is infinite if and only if $\tilde{\rho}$ contains an infinite family of states $\left\{\tau_{n}\right\}$ such that $\tau_{n}(I)=1,\left\|\tau_{n}-\tau_{m}\right\|=2$ if $n \neq m$, and $\tau_{n} \sim \tau_{m}$ for all $n$ and $m$.

The proofs of these theorems require the following results.

Theorem C. If $\rho$ is a state of the $C^{*}$-algebra $\mathfrak{A}$ then $\tau \in \tilde{\rho}$ if and only if $\phi_{r}$ is unitarily equivalent to a subrepresentation of $\phi_{\rho}$. Equivalently, $\tau \in \tilde{\rho}$ if and only if there exists a vector $x$ in $\mathcal{F C}_{\rho}$ such that $\tau=\omega_{x} \circ \phi_{\rho}$.

The nonalgebraic aspect of Theorem $\mathrm{C}$ depends on the following result.

Theorem D. If $\omega$ is a state of the $C^{*}$-algebra $\mathfrak{A}$ acting on the Hilbert space HC and $\left(x_{n}\right)$ is a sequence of vectors in $\mathcal{H C}$ such that $\| \omega-\omega_{x_{n}} \mid$ II $\| \rightarrow 0$, then $\omega$ is a vector state of $\mathfrak{A}$.

For the proof of this, we shall need:

Lemma $E$. If $R$ is a von Neumann algebra and $\left(E_{n}\right)$ is a sequence of cyclic projections in $R$ which tend strongly to $E$, then $E$ is a cyclic projection.

Proof. Let $F_{n}$ be the range projection of $E E_{n}$. Then $F_{n} \leqq E, F_{n}$ is cyclic in $a$, and $F_{n}$ tends strongly to $E$. In fact, with $x_{n}$ a unit vector generating $E_{n}$, $E x_{n}$ generates $F_{n}$; for $\left[\mathcal{R}^{\prime}\left(E x_{n}\right)\right]=\left[E \mathbb{R}^{\prime} x_{n}\right]=\left[E\left(E_{n}(\mathcal{H C})\right)\right]$, where $\mathcal{F C}$ is the underlying Hilbert space. With $x$ a unit vector in $E,\left(F_{n} x, x\right) \geqq\left(E E_{n} E x, x\right)$ $=\left(E E_{n} x, x\right) \rightarrow(x, x)=1$, since $E E_{n} E \leqq I$ and the range of $E E_{n} E$ is contained in $F_{n}$. Thus $F_{n} x \rightarrow x$.

Working with $E R E$ and $R^{\prime} E$ acting on $E(\mathcal{H C})$; we may assume that $E=I$. Note next that $I$ is countably decomposable. Indeed, if $\left\{G_{\alpha}\right\}$ is an orthogonal family of nonzero projections in $R$, let $G_{\alpha_{1}(n)}, G_{\alpha_{2}(n)}, \cdots$ be that countable subfamily of $\left\{G_{\alpha}\right\}$ consisting of those projections $G_{\alpha}$ such that $G_{\alpha} x_{n} \neq 0$. Then $\left\{G_{\alpha_{m}(n)}\right\}_{n, m}$ is a countable subfamily of $\left\{G_{\alpha}\right\}$; and if $G_{\alpha}$ is not in it, $G_{\alpha} x_{n}=0$, for all $n$. Thus $G_{\alpha} \mathcal{R}^{\prime} x_{n}=0$, and $G_{\alpha} E_{n}=0$, for all $n$. Since $E_{n}$ tends strongly to $I, G_{\alpha}=0 ;$ and $\left\{G_{\alpha}\right\}=\left\{G_{\alpha_{m}(n)}\right\}_{n, m}$. From [10, Lemma 3.3.7], we can choose a maximal cyclic projection $F$ in $R$ (one such that every other is $\lesssim$ it). If $P$ is a central projection such that $P F$ is purely infinite (in the sense of [10], i.e., $Q F$ is infinite for each nonzero central projection $Q \leqq P$ ); then $P F \sim P$, according to [10, Lemma 3.3.3]; so that $P$ is cyclic. Thus the purely infinite central portion of $F$ has cyclic central carrier; and it remains to prove that the central carrier, $Q$ of the finite portion of $F$ is cyclic. Restricting attention to $R Q$ and $R^{\prime} Q$ acting on $Q(\mathfrak{C})$, we may assume that $F$ is finite. Since $F$ is maximal cyclic, its central carrier is $I[10$, Lemma 3.3.6]. Thus, since $F$ is finite, 
$Q$ has no portion of type III. It follows that $F$ is contained in a properly larger finite projection unless $F=I$ (in which case, $I$ is cyclic and the proof is complete). Let $G$ be a finite projection containing $F$. As before, the range projections of $G E_{n}$ are cyclic, contained in $G$, and approach $G$ strongly. Restricting attention to $G R G$ and $R^{\prime} G$ acting on $G(\mathcal{H C})$, we may assume that $I(=G)$ is finite.

Let $x$ be a unit vector in $\mathcal{H}$. The state $A \rightarrow(\operatorname{Tr}(A) x, x)$ of $\mathbb{R}$ is completely additive, where $\operatorname{Tr}$ is the center-valued trace on $R[1$, Chapter III, $\$ 4 ; 2]$, in view of $[10$, Theorem 3.2.8(d)], since $\mathrm{Tr}$ is the dimension function on projections of $R$, and $R$ is countably decomposable. This state is, accordingly, normal (i.e., weakly continuous on the unit sphere of $R$ ) $[1$, Theorem 1, p. 54 and Example 9, p. 65]. Thus $(x, x) \geqq(\operatorname{Tr}(F) x, x) \geqq\left(\operatorname{Tr}\left(E_{n}\right) x, x\right) \rightarrow(x, x)=1$, since $E_{n} \precsim F \leqq I$. It follows that $\operatorname{Tr}(F)=I=F, I$ is cyclic (after the various reductions-or $E$ of the original notation is cyclic); and the proof is complete.

Proof of Theorem D. Note that with $\eta$ and $\tau$ normal states of $\mathfrak{A}-,\|\eta-\tau\|$ $=\|\eta|\mathfrak{A}-\tau| \mathfrak{A}\|$, for the unit ball of $\mathfrak{A}$ is strongly hence weakly dense in the unit ball of $\mathfrak{A}^{-}[13]$ and $\eta-\tau$ is weakly continuous on the unit ball of $\mathfrak{A}^{-}$. Thus $\left(\omega_{x_{n}} \mid \mathfrak{H}^{-}\right)$is a Cauchy convergent sequence. Its limit in the dual of $\mathfrak{A}^{-}$ is clearly a state of $\mathfrak{A}-$ and an extension of $\omega$ (which we denote again by $\omega$ ).

If $\epsilon>0$ is given, choose $n$ such that $\left\|\omega_{x_{n}}-\omega\right\|<\epsilon / 2$. Then, if $A$ in the unit ball of $\mathfrak{A}$ - is such that $\left|\omega_{x_{n}}(A)\right|=\left|\left(A x_{n}, x_{n}\right)\right|<\epsilon / 2,|\omega(A)|<\left|\omega_{x_{n}}(A)\right|+\epsilon / 2 \leqq \epsilon$. Thus $\omega$ is weakly continuous at 0 on the unit ball of $\mathfrak{A}^{-}$, and, by linearity, is weakly continuous on the entire unit ball, i.e., $\omega$ is normal on $\mathfrak{A}^{-}$.

Let $E_{n}$ be the support of $\omega_{x_{n}}$ in $\mathfrak{A}^{-}$and $E$ the support of $\omega$. We have $E_{n}=\left[\mathfrak{Y}^{\prime} x_{n}\right]$. Thus $F_{n}$, the closure of the range of $E E_{n}$, is a cyclic projection in $\mathfrak{U}^{-}$contained in $E$. In fact, $\left[\mathfrak{A}^{\prime} E x_{n}\right]=\left[E \mathfrak{A}^{\prime} x_{n}\right]=F_{n}$. With $a_{n}=\left\|\omega-\omega_{x_{n}}\right\|$;

$$
\begin{aligned}
\omega\left(E-F_{n}\right) & \leqq a_{n}+\omega_{x_{n}}\left(E-F_{n}\right)=a_{n}+\left(\left(E-F_{n}\right) x_{n}, x_{n}\right) \leqq a_{n}+\left\|\left(E-F_{n}\right) x_{n}\right\|^{2} \\
& =a_{n}+\left\|E E_{n} x_{n}-F_{n} x_{n}\right\|^{2}=a_{n}+\left\|F_{n}\left(E E_{n} x_{n}-x_{n}\right)\right\|^{2} \\
& \leqq a_{n}+\left\|E E_{n} x_{n}-x_{n}\right\|^{2}=a_{n}+\left((I-E) x_{n}, x_{n}\right)=a_{n}+\omega_{x_{n}}(I-E) \\
& \leqq a_{n}+a_{n}+\omega(I-E)=2 a_{n} .
\end{aligned}
$$

Thus $\omega\left(E-F_{n}\right) \rightarrow 0$. Now, from [1, Prop. 4, p. 62;3, Lemma 2.3$], F_{n}$ tends strongly to $E$. Whence, from Lemma $E, E$ is a cyclic projection in $\mathfrak{A}-$. If $y$ is a generating vector for $E, \omega_{y}$ and $\omega$ have $E$ as support. Thus $\omega$ is a vector state of $\mathfrak{A}-[1$, Chapter $I, \S 4]$.

Proof of Theorem C. Suppose, first, that $\tau=\omega_{x} \circ \phi_{\rho}$, for some vector $x$ in $\mathfrak{H}_{\rho}$. Since $\mathfrak{H}_{\rho}=\left[\phi_{\rho}(\mathfrak{H}) x_{\rho}\right]$, there is a sequence of operators $\left(A_{n}\right)$ in $\mathfrak{A}$ such that $\left\|\phi_{\rho}\left(A_{n}\right) x_{\rho}-x\right\| \rightarrow 0$. Now, if $B$ is in the unit ball of $\mathfrak{A}$, and $y, z$ are vectors in $\mathfrak{H}_{\rho},\left\|\phi_{\rho}(B)\right\| \leqq 1$, so that;

$$
\begin{aligned}
\left|\left(\phi_{\rho}(B) y, y\right)-\left(\phi_{\rho}(B) z, z\right)\right| & \leqq\left|\left(\phi_{\rho}(B) y, y-z\right)\right|+\left|\left(\phi_{\rho}(B)(y-z), z\right)\right| \\
& \leqq(\|y\|+\|z\|)\|y-z\| .
\end{aligned}
$$


Thus

$$
\left\|\omega_{\nu} \circ \phi_{\rho}-\omega_{z} \circ \phi_{\rho}\right\| \leqq(\|y\|+\|z\|)\|y-z\|
$$

In particular,

$$
\begin{aligned}
\left\|\rho_{A_{n}}-\tau\right\| & =\left\|\omega_{\phi_{\rho}\left(A_{n}\right) x_{\rho}} \circ \phi_{\rho}-\omega_{x} \circ \phi_{\rho}\right\| \\
& \leqq\left(\left\|\phi_{\rho}\left(A_{n}\right) x_{\rho}\right\|+\|x\|\right)\left(\left\|\phi_{\rho}\left(A_{n}\right) x_{\rho}-x\right\|\right) \\
& \leqq(2\|x\|+1)\left(\left\|\phi_{\rho}\left(A_{n}\right) x_{\rho}-x\right\|\right),
\end{aligned}
$$

for large $n$. Hence $\tau \in \tilde{\rho}$.

Suppose now that $\tau \in \tilde{\rho}$, so that there is a sequence of operators $\left(A_{n}\right)$ in $\mathfrak{A}$ such that $\left\|\rho_{\boldsymbol{A}_{\mathrm{n}}}-\tau\right\| \rightarrow 0$. Then $\tau$ annihilates the kernel of $\phi_{\rho}$, since each $\rho_{\boldsymbol{A}_{n}}$ does, and induces a linear functional $\tau^{\prime}$ of $\phi_{\rho}(\mathfrak{V})$. If $\phi_{\rho}(A) \geqq 0$ then $\phi_{\rho}\left(\left|A+A^{*}\right| / 2\right)=\phi_{\rho}(A)$ so that $\tau^{\prime}\left[\phi_{\rho}(A)\right]=\tau\left(\left|A+A^{*}\right| / 2\right) \geqq 0$, and $\tau^{\prime}$ is a state of $\phi_{\rho}(\mathfrak{U})$. Since $\phi_{\rho}$ is a continuous linear mapping of the Banach space $\mathfrak{A}$ onto the Banach space $\phi_{\rho}(\mathfrak{H})[25$, Corollary 3.1], it is an open mapping. By linearity then, for some $k>0$, the ball of radius $k$ about 0 in $\mathfrak{A}$ is mapped on to a set containing the unit ball in $\phi_{\rho}(\mathfrak{A})$ by $\phi_{\rho}$. With $T$ in $\phi_{\rho}(\mathfrak{U})$ and $\|T\| \leqq 1$, we may choose $A$ in $\mathfrak{A}$ with $\|A\| \leqq k$ such that $\phi_{\rho}(A)=T$. Thus

$$
\begin{aligned}
\left|\tau^{\prime}(T)-\omega_{\phi_{\rho}\left(A_{n}\right) x_{\rho}}(T)\right| & =\left|\tau^{\prime}\left[\phi_{\rho}(A)\right]-\left(\phi_{\rho}(A) \phi_{\rho}\left(A_{n}\right) x_{\rho}, \phi_{\rho}\left(A_{n}\right) x_{\rho}\right)\right| \\
& =\left|\tau(A)-\rho_{A_{n}}(A)\right| \leqq k\left\|\tau-\rho_{A_{n}}\right\| ;
\end{aligned}
$$

and $\left\|\tau^{\prime}-\omega_{\phi_{\rho}\left(A_{s}\right) x_{\rho}}\right\| \rightarrow 0$. From Theorem $D$, there is a vector $x$ in $\mathcal{H}_{\rho}$ such that $\tau^{\prime}=\omega_{x} \mid \phi_{\rho}(\mathfrak{I})$; so that $\tau=\omega_{x} \circ \phi_{\rho}$. With $E^{\prime}=\left[\phi_{\rho}(\mathfrak{I}) x\right]$, the subrepresentation $A \rightarrow \phi_{\rho}(A) E^{\prime}$ of $\phi_{\rho}$ is unitarily equivalent to $\phi_{\tau}$ (since each of these representations is cyclic with generating vectors for the images which induce the same state $\tau$ of $\mathfrak{A})$.

If $\phi_{\boldsymbol{r}}$ is unitarily equivalent to a subrepresentation of $\phi_{\rho}$ there is a vector $x$ in $\mathfrak{F}_{\rho}$ such that $\tau=\omega_{x} \circ \phi_{\rho}$; whence $\tau \in \tilde{\rho}$, from the first paragraph of this proof.

REMARK (3a). If $\rho$ is a pure state then, from [11], $\phi_{\rho}(\mathfrak{H})$ is $n$-fold transitive on $\mathfrak{H}_{\rho}$ so that $\tilde{\rho}=\left\{\rho_{A}: A\right.$ in $\left.\mathfrak{A}\right\}$, i.e., this last set is norm closed in the dual of I. Moreover, from [7, Corollary 8], $\tilde{\rho}=\left\{\rho_{U}: U\right.$ a unitary operator in $\left.\mathfrak{A}\right\}$.

Proof of Theorem A. Suppose that $\rho$ is a primary state of $\mathfrak{A}$ and $\tau, \eta \in \tilde{\rho}$. From Theorem $C$, there are vectors $x$ and $y$ in $\mathcal{K}_{\rho}$ such that $\tau=\omega_{x} \circ \phi_{\rho}$ and $\eta=\omega_{y} \circ \phi_{\rho}$. The projections $E^{\prime}=\left[\phi_{\rho}(\mathfrak{U}) x\right]$ and $F^{\prime}=\left[\phi_{\rho}(\mathfrak{A}) y\right]$ lie in the factor $\phi_{\rho}(\mathfrak{A})^{\prime}$ ( $\rho$ is primary). From [18, Lemma 6.2.3], $E^{\prime} \precsim F^{\prime}$ or $F^{\prime} \lesssim E^{\prime}$. Say $E^{\prime} \lesssim F^{\prime}$. A partial isometry in $\phi_{\rho}(\mathfrak{A})^{\prime}$ implementing this ordering effects a unitary equivalence between the representation $A \rightarrow \phi_{\rho}(A) E^{\prime}$ and a subrepresentation of $A \rightarrow \phi_{\rho}(A) F^{\prime}$. But these last representations are unitarily equivalent to $\phi_{\tau}$ and $\phi_{\eta}$, respectively (as noted at the end of the proof of Theorem C). Thus $\tau \in \tilde{\eta}$, in this case; that is, $\tau \lesssim \eta$. It follows that $\lesssim$ totally orders (the equivalence classes of) $\tilde{\rho}$. 
Suppose now that $\lesssim$ totally orders $\tilde{\rho}$; and let $P$ be a central projection in $\phi_{\rho}(\mathfrak{Y})^{\prime}$. Let $x=P x_{\rho}, y=(I-P) x_{\rho}, \tau=\omega_{x} \circ \phi_{\rho}$, and $\eta=\omega_{y} \circ \phi_{\rho}$. Suppose $\tau \lesssim \eta$. From Theorem $\mathrm{C}, \phi_{\tau}$ is unitarily equivalent to a subrepresentation of $\phi_{\eta}$; so that the representation $A \rightarrow \phi_{\rho}(A) P$ is unitarily equivalent to a subrepresentation of the representation $A \rightarrow \phi_{\rho}(A)(I-P)$ (since $P=\left[\phi_{\rho}(\mathfrak{U}) x\right]$ and $I-P$ $\left.=\left[\phi_{\rho}(\mathfrak{A}) y\right]\right)$. Let $U^{\prime}$ be a unitary operator which implements this equivalence, and define $V^{\prime}$ to be $U^{\prime}$ on $P$ and 0 on $I-P$. Then $V^{\prime}$ is a partial isometry in $\phi_{\rho}(\mathfrak{A})^{\prime}$ with initial space $P$ and final space in $I-P$. Thus $V^{\prime}=(I-P) V^{\prime} P=0$ and $P=0$. Symmetrically, the assumption $\eta \precsim \tau$ leads to the conclusion $I-P$ $=0$. Thus $\phi_{\rho}(\mathfrak{A})^{\prime}$ is a factor as is $\phi_{\rho}(\mathfrak{A})^{-}$; and $\rho$ is primary.

Note that $\rho$ is a minimal element relative to the partial ordering $\lesssim$ if and only if each subrepresentation of $\phi_{\rho}$ is unitarily equivalent to $\phi_{\rho}$ (from Theorem C); and this is the case if and only if each projection in $\phi_{\rho}(\mathfrak{A})^{\prime}$ is equivalent to $I$. Now, this situation obtains for projections in a von Neumann algebra if and only if it is a factor (since all projections are comparable) and it is countably-decomposable of type III (since all projections are cyclic and infinite) or it consists of scalar multiples of the identity operator (in this case, $\phi_{\rho}(\mathfrak{H})$ acts irreducibly and $\rho$ is pure). If we assume that $\mathfrak{K}_{\rho}$ is not one-dimensional, equivalently that $\rho / \rho(I)$ is not a homomorphism, and $\phi_{\rho}(\mathfrak{A})^{\prime}=\{a I\}$; then with $x$ and $y$ two linearly independent vectors in $\mathcal{H}_{\rho},(x, y)$ is a generating vector for $\mathfrak{H}_{\rho} \oplus \mathfrak{H}_{\rho}$ under $\left(\phi_{\rho} \oplus \phi_{\rho}\right)(\mathfrak{U})$ (since $\phi_{\rho}(\mathfrak{H})^{-}$is the algebra of all bounded operators on $\left.\mathfrak{H}_{\rho}[20]\right)$. Let $\tau=\omega_{(x, y)} \circ\left(\phi_{\rho} \oplus \phi_{\rho}\right)$. Now $\left(\phi_{\rho} \oplus \phi_{\rho}\right)(\mathfrak{A})^{\prime}$ is a factor of type $I_{2}$; so that $E^{\prime}=\left[\left(\phi_{\rho} \oplus \phi_{\rho}\right)(\mathfrak{A})(x, 0)\right]$ is not equivalent to $I$. But $\phi_{\rho}$ is unitarily equivalent to $A \rightarrow\left(\phi_{\rho} \oplus \phi_{\rho}\right)(A) E^{\prime}$. Thus $\rho<\tau$ and $\rho$ is not maximal in the set of primary states.

We show, next, that $\rho$ is maximal in the set of primary states if and only if $\phi_{\rho}(\mathfrak{H})^{\prime}$ is infinite or $\phi_{\rho}(\mathfrak{H})^{\prime}$ has a cyclic vector (so that $\phi_{\rho}(\mathfrak{H})^{-}$and $\phi_{\rho}(\mathfrak{H})^{\prime}$ have coupling 1). In fact, if $\phi_{\rho}(\mathfrak{H})^{\prime}$ is infinite, and $\rho \precsim \tau$; then $\left[\phi_{\tau}(\mathfrak{H}) x\right]$ $\sim\left[\phi_{\tau}(\mathfrak{H}) x_{\tau}\right]=\mathfrak{H C}_{\tau}$, where $x$ in $\mathfrak{H C}_{\tau}$ satisfies $\omega_{x} \circ \phi_{\tau}=\rho$ (cf. Theorem $\mathrm{C}$ for the existence of such an $x$ ), since $\left[\phi_{\tau}(\mathfrak{A}) x\right]$ and $\mathfrak{H}_{\tau}$ are infinite cyclic projections in the factor $\phi_{\tau}(\mathfrak{H})^{\prime}[10$, Lemma 3.3.3]. Thus $\rho$ is a maximal state in the set of primary states.

Suppose $\phi_{\rho}(\mathfrak{U})^{\prime}$ is not infinite but has a cyclic vector, and let $\tau$ be a primary state of $\mathfrak{A}$ such that $\rho \precsim \tau$. Choose $x$ in $\mathfrak{H C}_{\tau}$ such that $\omega_{x} \circ \phi_{\tau}=\rho$. Since $\phi_{\rho}$ is unitarily equivalent to $A \rightarrow \phi_{\tau}(A) E^{\prime}$, where $E^{\prime}=\left[\phi_{\tau}(\mathfrak{A}) x\right] ; E^{\prime} \phi_{\tau}(\mathfrak{H})^{\prime} E^{\prime}$ is finite, $\phi_{\tau}(\mathfrak{A})^{-} E^{\prime}$ is finite since $\phi_{\rho}(\mathfrak{U})^{-}$is $[18$, Theorem $\mathrm{X}]$, and $\left[E^{\prime} \phi_{\tau}(\mathfrak{U})^{\prime} E^{\prime} x\right]$ $=E^{\prime}\left[\phi_{\tau}(\mathfrak{A})^{\prime} x\right]=E^{\prime}$ (since $\phi_{\tau}(\mathfrak{A})^{-} E^{\prime}$ is finite with cyclic vector $x$ for $E^{\prime}$, and $E^{\prime} \phi_{\tau}(\mathfrak{A})^{\prime} E^{\prime}$ has some cyclic vector). Now $\left[\phi_{\tau}(\mathfrak{U})^{\prime} x\right]$ lies in $\phi_{\tau}(\mathfrak{U})^{-}$, a factor; whence, from $E^{\prime}\left[\phi_{\tau}(\mathfrak{A})^{\prime} x\right]=E^{\prime}$ and $[18$, Theorem III $],\left[\phi_{\tau}(\mathfrak{A})^{\prime} x\right]=I$. Thus $\left[\phi_{\tau}(\mathfrak{A}) x\right]=E^{\prime}$ is maximal cyclic in $\phi_{\tau}(\mathfrak{H})^{\prime}[10, \mathrm{p} .340]$; and, since $\mathfrak{H}_{\tau}$ is cyclic under $\phi_{\tau}(\mathfrak{A})^{-}, E^{\prime}$ is equivalent to $I$ in $\phi_{\tau}(\mathfrak{A})^{\prime}$. Thus $\rho \sim \tau$, and $\rho$ is maximal in the set of primary states.

If $\phi_{\rho}(\mathfrak{A})^{\prime}$ is finite and is not cyclic, then $\left(\phi_{\rho} \oplus \phi_{\rho}\right)(\mathfrak{U})^{\prime}$ is the tensor product 
of $\phi_{\rho}(\mathfrak{A})^{\prime}$ with a factor of type $\mathrm{I}_{2}$ and is, therefore, finite. With $E^{\prime}=\left[\left(\phi_{\rho} \oplus \phi_{\rho}\right)(\mathfrak{H})\left(x_{\rho}, 0\right)\right], A \rightarrow\left(\phi_{\rho} \oplus \phi_{\rho}\right)(A) E^{\prime}$ is unitarily equivalent to $\phi_{\rho}$. Let $F^{\prime}$ be a maximal cyclic projection in $\left(\phi_{\rho} \oplus \phi_{\rho}\right)(\mathfrak{A})^{\prime}$ containing $E^{\prime}$ [10, Lemma 3.3.7]. If $E^{\prime}<F^{\prime}$ then, by finiteness, $E^{\prime}$ is not equivalent to $F^{\prime}$; and the cyclic representation, $A \rightarrow\left(\phi_{\rho} \oplus \phi_{\rho}\right)(A) F^{\prime}$ is not unitarily equivalent to $\phi_{\rho}$. Thus $\rho$ is not maximal in the set of primary states. We may assume, therefore, that $E^{\prime}$ is maximal cyclic in $\left(\phi_{\rho} \oplus \phi_{\rho}\right)(\mathfrak{A})^{\prime}$. From its definition, $E^{\prime}$ is not $\mathfrak{H}_{\rho} \oplus \mathfrak{K}_{\rho}$; whence, by finiteness and maximal cyclicity of $E^{\prime}, \mathfrak{H C}_{\rho} \oplus \mathfrak{K C}_{\rho}$ is not cyclic under $\left(\phi_{\rho} \oplus \phi_{\rho}\right)(\mathfrak{A})$. Thus, from [8, Lemma 1.2.8; 10, Lemma 3.3.6], $\mathfrak{H C}_{\rho} \oplus \mathfrak{F C}_{\rho}$ is cyclic under $\left(\phi_{\rho} \oplus \phi_{\rho}\right)(\mathfrak{R})^{\prime}$. Since $E^{\prime}$ is maximal cyclic in $\left(\phi_{\rho} \oplus \phi_{\rho}\right)(\mathfrak{H})^{\prime}$, some generator $x$ for $E^{\prime}$ generates $\mathfrak{H C}_{\rho} \oplus \mathfrak{H C}_{\rho}$ under $\left(\phi_{\rho} \oplus \phi_{\rho}\right)(\mathfrak{R})^{\prime}$; so that $\left(\phi_{\rho} \oplus \phi_{\rho}\right)(\mathfrak{H})^{-} E^{\prime}$ and $E^{\prime}\left(\phi_{\rho} \oplus \phi_{\rho}\right)(\mathfrak{R})^{\prime} E^{\prime}$ acting on $E^{\prime}\left(\mathfrak{H C}_{\rho} \oplus \mathfrak{H}_{\rho}\right)$ have a joint generating vector. However, these von Neumann algebras acting on $E^{\prime}\left(\mathfrak{F C}_{p} \oplus \mathfrak{H C}_{p}\right)$ are unitarily equivalent to $\phi_{\rho}(\mathfrak{H})^{-}, \phi_{\rho}(\mathfrak{H})^{\prime}$ acting on $\mathfrak{H C}_{p}$; so that they too have a joint generating vector, contrary to assumption. Thus $E^{\prime}$ is not maximal cyclic and $\rho$ is not maximal in the set of primary states.

These characterizations of minimal states and maximal primary states leave only the type I description to be established. Now, $\rho$ is primary and of type I if and only if $\phi_{\rho}(\mathfrak{A})^{\prime}$ is a type I factor [18, Lemma 5.3.1]. The factor $\phi_{\rho}(\mathfrak{H})^{\prime}$ is of type I if and only if it contains a minimal projection $E^{\prime}$. With $x$ a generator for $E^{\prime}$, the state $\tau=\omega_{x} \circ \phi_{\rho}$ in $\tilde{\rho}$ is pure if and only if the representation $A \rightarrow \phi_{\rho}(A) E^{\prime}$ is irreducible, which is the case if and only if $E^{\prime}$ is minimal in $\phi_{\rho}(\mathfrak{R})^{\prime}$.

Remarks (3b). Two states $\tau$ and $\rho$ of $\mathfrak{A}$ are disjoint if and only if their representations are disjoint. In fact $\tilde{\tau}$ and $\tilde{\rho}$ contain $\eta$ if and only if there are vectors $x$ and $y$ in $\mathfrak{K}_{\tau}$ and $\mathfrak{H}_{\rho}$, respectively, such that $\eta=\omega_{x} \circ \phi_{\tau}=\omega_{y} \circ \phi_{\rho}$, from Theorem C. For such vectors, the subrepresentations, $A \rightarrow \phi_{\tau}(A) E^{\prime}$ and $A \rightarrow \phi_{\rho}(A) F^{\prime}$, where $E^{\prime}=\left[\phi_{r}(\mathfrak{H}) x\right]$ and $F^{\prime}=\left[\phi_{\rho}(\mathfrak{A}) y\right]$, of $\phi_{\tau}$ and $\phi_{\rho}$, respectively, are unitarily equivalent. Conversely, if there are unitarily equivalent subrepresentations of $\phi_{r}$ and $\phi_{\rho}$, with $x$ and $y$ unit vectors carried onto one another by a unitary transformation implementing this equivalence, then $\omega_{x} \circ \phi_{\tau}=\omega_{y} \circ \phi_{\rho}$ is contained in $\tilde{\tau}$ and $\tilde{\rho}$, from Theorem C.

(3c). Combining (3b) with the criterion of Theorem A for $\rho$ to be primary, it follows that $\rho$ is primary if and only if $\tilde{\rho}$ does not contain nonzero disjoint states. In fact if $\precsim$ totally orders $\tilde{\rho}$, this is clearly the case. On the other hand, if $\rho$ is not primary and $P$ is a central projection in $\phi_{\rho}(\mathfrak{A})^{\prime}$ such that $0 \neq P \neq I$; then, as in the second paragraph of the proof of Theorem A, $A \rightarrow \phi_{\rho}(A) P$ and $A \rightarrow \phi_{\rho}(A)(I-P)$ are disjoint representations. With $x$ and $y$ unit vectors in $P$ and $I-P$, respectively, the states $\omega_{x} \circ \phi_{\rho}$ and $\omega_{y} \circ \phi_{\rho}$ in $\tilde{\rho}$, from Theorem C, are disjoint, by (3b) above.

(3d). In the statement and throughout the proof of Theorem A, we have left the meaning of minimality of $\rho$ somewhat ambiguous. This causes no difficulty since the two reasonable possibilities, "minimal in the set of states" 
and "minimal in the set of primary states" coincide. In fact, if $\rho$ is minimal in the set of all states, we have the situation dealt with in the proof of Theorem $A ; \rho$ is primary and, of course, minimal in the set of primary states. If $\rho$ is minimal in the set of primary states and $\tau \precsim \rho$, then $\tau$ is primary, from Theorem A, so that $\tau \sim \rho$ and $\rho$ is minimal in the set of all states.

The situation is quite different with regard to maximality. We shall discuss this in detail in the next section (cf. Theorem $\mathrm{H}$ ).

For the proof of Theorem B, we shall need:

Lemma F. If $\rho$ is a state of the $C^{*}$-algebra $\mathfrak{A}, \tau$ and $\eta$ lie in $\tilde{\rho}$, and $\tau^{\prime}, \eta^{\prime}$ are normal states of $\phi_{\rho}(\mathfrak{U})^{-}$such that $\tau=\tau^{\prime} \circ \phi_{\rho}, \eta=\eta^{\prime} \circ \phi_{\rho}$; then the carriers of $\tau^{\prime}$ and $\eta^{\prime}$ are orthogonal if and only if $\|\tau-\eta\|=\|\tau\|+\|\eta\|$.

Proof. We may suppose that $\mathfrak{A}$ acting on $\mathfrak{H}$ is the universal representation of $\mathfrak{A}$ (the direct sum of cyclic representations of $\mathfrak{A}$ corresponding to the distinct states of $\mathfrak{X})$. Assuming this, there is a vector $x$ in $\mathcal{H}$ such that $\omega_{x} \mid \mathfrak{A}=\rho$ and vectors $y$ and $z$ in $E^{\prime}$ such that $\omega_{\nu} \mid \mathfrak{A}=\tau$ and $\omega_{z} \mid \mathfrak{A}=\eta$, where $E^{\prime}=[\mathfrak{A} x]$. From [13], $\|\tau-\eta\|=\left\|\left(\omega_{y}-\omega_{z}\right) \mid \mathfrak{A}-\right\|=\|\tau\|+\|\eta\|=\|y\|^{2}+\|z\|^{2}$.

The carrier of $\omega_{y} \mid \mathfrak{A}-$ is $\left[\mathfrak{U}^{\prime} y\right]$ and that of $\omega_{z} \mid \mathfrak{A}-$ is [ $\left.\mathfrak{U}^{\prime} z\right]$. Identifying $\phi_{\rho}$ with $A \rightarrow A E^{\prime}$ (they are unitarily equivalent representations of $\mathfrak{U}$ ), the carrier of $\tau^{\prime}$ is $\left[E^{\prime} \mathfrak{X}^{\prime} E^{\prime} y\right] \subseteq\left[\mathfrak{H}^{\prime} y\right]$ and that of $\eta^{\prime}$ is $\left[E^{\prime} \mathfrak{A}^{\prime} E^{\prime} z\right] \subseteq\left[\mathfrak{A}^{\prime} z\right]$. It will suffice, therefore, to establish the special case: $\left\|\left(\omega_{y}-\omega_{z}\right) \mid \propto\right\|=\|y\|^{2}+\|z\|^{2}$, for a von Neumann algebra $R$, implies that $\left[\mathcal{R}^{\prime} y\right]$ and $\left[\mathcal{R}^{\prime} z\right]$ are orthogonal.

Let $G$ be the space generated by $y$ and $z$. Since $\left(\omega_{y}-\omega_{z}\right)(A)=\left(\omega_{y}-\omega_{z}\right)(G A G)$ and $\|G A G\| \leqq\|A\|$, as a functional on $G R G, \omega_{y}-\omega_{z}$ has norm $\|y\|^{2}+\|z\|^{2}$. Let $A$ be an operator of norm 1 in $G R G$ such that $\left(\omega_{y}-\omega_{z}\right)(A)=(A y, y)-(A z, z)$ $=\|y\|^{2}+\|z\|^{2}$. From the Schwarz inequality, $(A y, y)=\|y\|^{2}$ and $(A z, z)$ $=-\|z\|^{2}$. From the Schwarz equality, $A y=y$ and $A z=-z$. With $\left(A+A^{*}\right) / 2$ in place of $A$, we conclude that $y$ and $z$ are eigenvectors of a self-adjoint operator corresponding to different eigenvalues; whence $y$ and $z$ are orthogonal.

With $U^{\prime}$ a unitary operator in $R^{\prime}, \omega_{y}\left|R=\omega_{U^{\prime} y}\right| R$, and $\omega_{z}\left|R=\omega_{U^{\prime}}\right| R$. From the above, $U^{\prime} y$ and $V^{\prime} z$ are orthogonal for each pair of unitary operators $U^{\prime}, V^{\prime}$ in $\mathcal{R}^{\prime}$. Now the unitary operators generate $R^{\prime}$ linearly $[1$, p. 4$]$; so that $\left[R^{\prime} y\right]$ and $\left[R^{\prime} z\right]$ are orthogonal, and the proof of the sufficiency is complete.

To prove the necessity, we note that if the carriers $G$ and $F$ of $\eta^{\prime}$ and $\tau^{\prime}$, respectively, are orthogonal, then $\left(\tau^{\prime}-\eta^{\prime}\right)(F-G)=\left\|\tau^{\prime}\right\|+\left\|\eta^{\prime}\right\|=\tau^{\prime}(I)+\eta^{\prime}(I)$ $=\|\tau\|+\|\eta\|$. With $A$ in $\mathfrak{A}^{-}$such that $A E^{\prime}=F-G,\left\|A E^{\prime}\right\|=\|F-G\|=1$, $\tau^{\prime}\left(A E^{\prime}\right)=(A y, y)$, and $\eta^{\prime}\left(A E^{\prime}\right)=(A z, z)$. The mapping $A E^{\prime} \rightarrow A P$, where $P$ is the central carrier of $E^{\prime}$, is a * isomorphism of $\mathfrak{A}-E^{\prime}$ onto $\mathfrak{A}-P[10$, Corollary 3.1.2]. Thus $\left\|A E^{\prime}\right\|=\|A P\|=1[5]$, and, since $y$ and $z$ are in $E^{\prime}(\leqq P)$, $\left(\omega_{y}-\omega_{z}\right)(A P)=(A P y, y)-(A P z, z)=(A y, y)-(A z, z)=\|\tau\|+\|\eta\|$ $\leqq\left\|\left(\omega_{\nu}-\omega_{z}\right) \mid \mathfrak{Q}-\right\|=\|\tau-\eta\|$. Of course, $\|\tau-\eta\| \leqq\|\tau\|+\|\eta\|$; so that $\|\tau-\eta\|=\|\tau\|$ $+\|\eta\|$, and the proof is complete. 
Proof of Theorem B. The algebra $\phi_{\rho}(\mathfrak{R})-$ is infinite if and only if it contains an infinite number of equivalent orthogonal nonzero cyclic projections $\left\{E_{n}\right\}$. With $x_{n}$ a unit generating vector for $E_{n}$, let $\tau_{n}=\omega_{x_{n}} \circ \phi_{\rho}$. Then $\tau_{n}(I)=1$, $\left\|\tau_{n}-\tau_{m}\right\|=2$, when $n \neq m$ (from Lemma F), $\tau_{n} \in \tilde{\rho}, \tau_{n} \sim \tau_{m}$, since $E_{n}$ and $E_{m}$ are equivalent in $\phi_{\rho}(\mathfrak{A})^{-}$, so that $\left[\phi_{\rho}(\mathfrak{A}) x_{n}\right]$ and $\left[\phi_{\rho}(\mathfrak{A}) x_{m}\right]$ are equivalent in $\phi_{\rho}(\mathfrak{A})^{\prime}[18$, Lemma 9.3.3].

If there is an infinite set $\left\{\tau_{n}\right\}$ of nonzero states in $\tilde{\rho}$ with $\left\|\tau_{n}-\tau_{m}\right\|=\left\|\tau_{n}\right\|$ $+\left\|\tau_{m}\right\|$, for $n \neq m$, and such that $\tau_{n} \sim \tau_{m}$; then $\phi_{\rho}(\mathfrak{A})-$ is infinite. In fact, with $x_{m}$ a vector in $\mathfrak{F}_{\rho}$ such that $\tau_{n}=\omega_{x_{n}} \circ \phi_{\rho}$, the carriers of $\left\{\omega_{x_{n}} \mid \phi_{\rho}(\mathfrak{R})^{-}\right\}$form an orthogonal family of projections in $\phi_{\rho}(\mathfrak{R})^{-}$, from Lemma $F$. These carriers $\left\{\left[\phi_{\rho}(\mathfrak{R})^{\prime} x_{n}\right]\right\}$ are equivalent, from [18, Lemma 9.3.3], for $\left\{\left[\phi_{\rho}(\mathfrak{A}) x_{n}\right]\right\}$ are equivalent (since $\left\{\tau_{n}\right\}$ are equivalent).

4. Further developments. The order $\lesssim$ on $\tilde{\rho}$ relates to the Murray-von Neumann ordering of the projections in $\phi_{\rho}(\mathfrak{A})^{\prime}$. The ordering imposed by $\bar{\tau} \subseteq \bar{\eta}$ (cf. Definition 2.1) on the states of $\tilde{\rho}$ is associated with the standard operator ordering of the cyclic projections in $\phi_{\rho}(\mathfrak{R})^{-}$. We have:

Theorem G. If $\rho$ is a state of the $C^{*}$-algebra $\mathfrak{A}$ then $\bar{\rho}=\left\{\omega_{x} \circ \phi_{\rho}: x\right.$ a vector in the carrier of $\left.\omega_{x_{\rho}} \mid \phi_{\rho}(\mathfrak{R})^{-}\right\}$.

Proof. Note first that the carrier of $\omega_{x_{\rho}} \mid \phi_{\rho}(\mathfrak{R})^{-}$is $\left[\phi_{\rho}(\mathfrak{A})^{\prime} x_{\rho}\right]=E$; and that, with $y=A^{\prime} x_{\rho}, A^{\prime}$ in $\phi_{\rho}(\mathfrak{R})^{\prime}, \omega_{y} \circ \phi_{\rho} \leqq\left\|A^{\prime}\right\|^{2} \rho$ (since $\left.A^{\prime *} A^{\prime} \leqq\left\|A^{\prime}\right\|^{2} I\right)$. Moreover, from the estimate of the first paragraph in the proof of Theorem C; with $x$ in $\left[\phi_{\rho}(\mathfrak{A})^{\prime} x_{\rho}\right], \omega_{x} \circ \phi_{\rho}$ is a norm limit of states of $\mathfrak{A}$ majorized by some multiple of $\rho$ (i.e., in $\bar{\rho}$ ).

If $\tau$ is in $\bar{\rho}$ and $A$ is in the kernel of $\phi_{\rho}$, then $\phi_{\rho}\left(A^{*} A\right)=0$; so that $0=\rho\left(A^{*} A\right)$ $=\eta\left(A^{*} A\right)$, for each state $\eta$ of $\mathfrak{A}$ majorized by a multiple of $\rho$. Thus $\tau\left(A^{*} A\right)=0$ for norm limits $\tau$ of states such as $\eta$; and, from the Schwarz inequality, $\tau(A)=0$. It follows, as in the proof of Theorem C, that there is a state $\tau^{\prime}$ of $\phi_{\rho}(\mathfrak{R})$ such that $\tau^{\prime} \circ \phi_{\rho}=\tau$. With $\eta$ a state of $\mathfrak{A}$ such that $0 \leqq \eta \leqq a \rho$, and $\phi_{\rho}(A) \geqq 0$ (we may assume that $A \geqq 0$, as in the proof of Theorem C), $0 \leqq \eta(A)$ $=\left(\eta^{\prime} \circ \phi_{\rho}\right)(A) \leqq a \rho(A)=a\left(\omega_{x \rho} \circ \phi_{\rho}\right)(A)$; whence $0 \leqq \eta^{\prime} \leqq a \omega_{x_{\rho}} \mid \phi_{\rho}(\mathfrak{R})$. From [3, Lemma 2.2], $\eta^{\prime}=\omega_{A^{\prime} x_{\rho}}$ with $A^{\prime}$ in $\phi_{\rho}(\mathfrak{R})^{\prime}$. As in the proof of Theorem $\mathrm{C}, \tau^{\prime}$ is a norm limit of states $\eta^{\prime}$ of $\phi_{\rho}(\mathfrak{R})$. From Theorem $\mathrm{D}, \tau^{\prime}=\omega_{z} \mid \phi_{\rho}(\mathfrak{R})$; so that $\tau=\omega_{z} \circ \phi_{\rho}$. Now, with $\eta^{\prime}=\omega_{A^{\prime} x_{\rho}}\left|\phi_{\rho}(\mathfrak{R}),\left\|\eta^{\prime}-\tau^{\prime}\right\|=\left\|\omega_{A^{\prime} x_{\rho}}\left|\phi_{\rho}(\mathfrak{R})^{-}-\omega_{z}\right| \phi_{\rho}(\mathfrak{R})-\right\|\right.$, and $\left(E A^{\prime} x_{\rho}, A^{\prime} x_{\rho}\right)=\left\|A^{\prime} x_{\rho}\right\|^{2}$. Thus $(E z, z)=\|z\|^{2}$ (using $\left.\eta^{\prime}(I) \rightarrow \tau^{\prime}(I)\right)$; and $z$ lies in $E$. This completes the proof.

ReMARK (4a). The proof above establishes, as well, that with $\tau\left(=\omega_{y} \circ \phi_{\rho}\right)$ in $\tilde{\rho}, \bar{\tau}=\left\{\omega_{x} \circ \phi_{\rho}: x\right.$ a vector in the carrier of $\left.\omega_{y} \mid \phi_{\rho}(\mathfrak{R})^{-}\right\}$. If $E$ is the carrier of $\omega_{y} \mid \phi_{\rho}(\mathfrak{R})^{-}$, it is equally true that $\bar{\tau}$ consists of the states induced by the vectors of $E E^{\prime}$, where $E^{\prime}=\left[\phi_{\rho}(\mathfrak{R}) y\right]$. The last line of the proof of Theorem $\mathrm{G}$, also proves that if $\omega_{z} \circ \phi_{\rho}$ is in $\bar{\tau}$, then $z$ is in $E$.

The identifications made by Theorems $\mathrm{C}$ and $\mathrm{G}$ of $\tilde{\rho}$, and $\bar{\tau}$, respectively, yield $\bar{\rho} \subseteq \tilde{\rho}$. 
DeFinition 4.1. We call a state $\rho$ of $\mathfrak{A}$ separating when $\overline{\boldsymbol{\rho}}=\tilde{\boldsymbol{\rho}}$.

As an easy consequence of Theorems $C$ and $G$, we have:

Corollary. A state $\rho$ of a $C^{*}$-algebra $\mathfrak{A}$ is separating if and only if $x_{\rho}$ is a separating vector for $\phi_{\rho}(\mathfrak{A})^{-}$.

Proof. The vector $x_{\rho}$ is separating for $\phi_{\rho}(\mathfrak{H})^{-}$if and only if it is cyclic for $\phi_{\rho}(\mathfrak{U})^{\prime}$. This is the case if and only if $\left[\phi_{\rho}(\mathfrak{U})^{\prime} x_{\rho}\right]=\mathfrak{H C}_{\rho}=\left[\phi_{\rho}(\mathfrak{U}) x_{\rho}\right]$. From Theorems $\mathrm{C}, \mathrm{G}$, and the remark following, this last is equivalent to $\tilde{\rho}=\bar{\rho}$.

Each state $\tau$ of $\mathfrak{A}$ in $\tilde{\rho}$ corresponds to an equivalence class of projections in $\phi_{\rho}(\mathfrak{A})^{\prime}$, the cyclic projections generated by vectors $y$ in $\mathfrak{F}_{\rho}$ such that $\tau=\omega_{y} \circ \phi_{\rho}$ (i.e., the projections in $\phi_{\rho}(\mathfrak{H})^{\prime}$ restrictions to which give representations unitarily equivalent to $\phi_{\tau}$ ). On the other hand, the same vectors $y$ all generate a single projection in $\phi_{\rho}(\mathfrak{U})^{-}$, the carrier of the normal extension of $\tau^{\prime}$ from $\phi_{\rho}(\mathfrak{A})$ to $\phi_{\rho}(\mathfrak{A})^{-}$(using the notation of the proof of Theorem $G$ ). For the remainder, we adopt the notation $\tau^{\prime}$ for the normal state of $\phi_{\rho}(\mathfrak{U})^{-}$such that $\tau=\tau^{\prime} \circ \phi_{\rho}$.

Referring to the foregoing association of states in $\tilde{\rho}$ with projections in $\phi_{\rho}(\mathfrak{U})^{-}$and $\phi_{\rho}(\mathfrak{U})^{\prime}$, we can develop a dimension theory on $\tilde{\rho}$, in terms of the states of $\mathfrak{A}$, which will reflect the dimension theories of $\phi_{\rho}(\mathfrak{A})^{-}$and $\phi_{\rho}(\mathfrak{A})^{\prime}$. The details are routine from the comments which follow. With $\tau$ and $\eta$ in $\tilde{\rho}$, orthogonality of the carriers of $\tau^{\prime}$ and $\eta^{\prime}$ is equivalent to $\|\tau-\eta\|=\|\tau\|+\|\eta\|$, from Lemma F. Using [18, Lemma 9.3.3], equivalence of the carriers of $\tau^{\prime}$ and $\eta^{\prime}$ is the same as $\tau \sim \eta$. Having equivalence and orthogonality, the dimension theory on $\tilde{\rho}$ can now be constructed in any of the usual ways (e.g. [10, $\S 2 ; 18$, Chapter VIII] ). This will correspond to the dimension theory of the cyclic projections in $\phi_{\rho}(\mathfrak{H})^{-}$. In view of the coupling theory $[8 ; 9 ; 18]$ and the fact that all projections in $\phi_{\rho}(\mathfrak{A})^{\prime}$ are cyclic, this theory will correspond to the full dimension theory on these projections, once we have described the coupling in terms of $\tilde{\rho}$. For this, we use Zorn's Lemma to locate a maximal set of states $\left\{\tau_{\gamma}\right\}$ in $\tilde{\rho}$ each equivalent to $\rho$ and orthogonal in the above sense. The number of these states together with the dimension of a state $\tau$ in $\tilde{\rho}$ of maximal dimension orthogonal to each $\tau_{\gamma}$ (normalizing so that $\rho$ has dimension 1 in case it is finite) describes the coupling.

We take up again the question of maximal elements in the state space of $\mathfrak{A}$ (as opposed to maximal elements in the set of primary states; $\mathrm{cf}$. the proof of Theorem A and Remark (3d)). While there will exist states which are maximal in the set of primary states, in general there are no states which are maximal in the set of all states (equivalently, no maximal cyclic representations). In fact, we prove:

Theorem H. If $\mathfrak{A}$ is a countably generated $C^{*}$-algebra, then the following conditions are equivalent:

(a) $\mathfrak{A}$ has a maximal state. 
(b) $\mathfrak{A}$ has at most a countable number of unitarily inequivalent irreducible representations.

(c) $\mathfrak{A}$ is a type I $C^{*}$-algebra with countable structure space (i.e., a countable number of primitive ideals).

(d) $\mathfrak{A}$ is a GCR algebra with countable structure space.

Proof. (a) $\rightarrow$ (b): Suppose $\rho$ is a maximal element and $\tau$ any element of the state space of $\mathfrak{A}$. With $\phi=\phi_{\rho} \oplus \phi_{\tau}, \mathfrak{F}=\mathfrak{F C}_{\rho} \oplus \mathfrak{K C}_{\tau}$, and $E_{\rho}$ and $E_{\tau}$ the orthogonal projections of $\mathfrak{H C}$ onto $\mathfrak{H C}_{\rho}$ and $\mathfrak{H C}_{\tau}$, respectively, then $E_{\rho}$ and $E_{\tau}$ are in $\phi(\mathfrak{U})^{\prime}$. Let $P$ and $Q$ be the central carriers of $E_{\rho}$ and $E_{\tau}$, respectively. Since $\rho$ is maximal, $E_{\rho}$ is a maximal cyclic projection in $\phi(\mathfrak{A})^{\prime}$ (in the sense of $[10$, p. 340]). From the remarks preceding [10, Lemma 3.3.6], all maximal cyclic projections in $\phi(\mathfrak{A})^{\prime}$ are equivalent. From [10, Lemma 3.3.7], $E_{\tau}$ is contained in some maximal cyclic projection; so that $E_{\tau} \precsim E_{\rho}$, and $\tau \lesssim \rho$. It follows that each state of $\mathfrak{A}$ lies in $\tilde{\rho}$, and $\rho$ (really, $\tilde{\rho}$ ) is the maximal element.

From Theorem $\mathrm{C}$, each state of $\mathfrak{A}$ has the form $\omega_{x} \circ \phi_{\rho}$, with $x$ a vector in $\mathfrak{H}_{\rho}$. Since the states of $\mathfrak{A}$ form a separating family, $\phi_{\rho}$ is an isomorphism. The vector $x_{\rho}$ is separating for $\phi_{\rho}(\mathfrak{U})^{\prime}$, so that $\phi_{\rho}(\mathfrak{A})^{\prime}$ and its center are countably decomposable. Since each state of $\mathfrak{A}$ arises from a vector in $\mathfrak{H}_{\rho}$, each cyclic representation of $\mathfrak{A}$ is unitarily equivalent to the restriction of $\phi_{\rho}$ to a cyclic projection in $\phi_{\rho}(\mathfrak{A})^{\prime}$. Disjoint representations correspond to projections in $\phi_{\rho}(\mathfrak{A})^{\prime}$ with mutually orthogonal central carriers. Now, unitarily inequivalent irreducible representations are disjoint. Each such representation of $\mathfrak{A}$ is a subrepresentation of $\phi_{\rho}$, and $\phi_{\rho}(\mathfrak{U})^{\prime}$ has a countably decomposable center. Thus $\mathfrak{A}$ has at most a countable number of unitarily inequivalent irreducible representations. This implication is valid for arbitrary $C^{*}$-algebras.

(b) $\rightarrow$ (c): Suppose now that $\mathfrak{A}$ is countably generated as a $C^{*}$-algebra (i.e., $\mathfrak{A}$ is the smallest $C^{*}$-subalgebra containing some countable subset). Equivalently, we may assume that some countable subset (the polynomials with rational coefficients in the generators) is norm dense in $\mathfrak{A}$. Thus each cyclic representation of $\mathfrak{A}$ occurs on a separable space. In essence, the results of $[17$, Theorem $3.1 ; 22]$, making use of the deeper portions of reduction theory [21] complete the proof of this part. In fact, using [1, Theorem 2, p. 220, Corollary, p. 169, Corollary 1(i), p. 179, Proposition 6, p. 163, Theorem $3(\mathrm{a})$, p. 174], we conclude, by reducing a cyclic representation of $\mathfrak{A}$ relative to a maximal abelian subalgebra of its commutant, that the commutant of each such representation contains a minimal projection. Of course, the central carrier of a minimal projection is a minimal central projection; and restriction to it is a factor representation of type I. By the same token, the orthogonal complement of this minimal central projection contains a minimal projection. Thus, the commutant of the image is a direct sum of factors of type I. It follows that the $C^{*}$-algebra $\mathfrak{A}$ is a type I algebra.

We give a proof of $(b) \rightarrow(c)$ which establishes $(b) \rightarrow(a)$ and which does not appeal to reduction theory. From each unitary equivalence class of 
irreducible representations of $\mathfrak{A}$, choose one representation $\phi_{n}$ of $\mathfrak{A}$ as operators on $\mathcal{H}_{n}$. Let $\psi_{n}$ be the representation of $\mathfrak{A}$ on $\mathfrak{H C}_{n} \otimes \mathcal{H}_{n}$ defined by: $\psi_{n}(A)$ $=\phi_{n}(A) \otimes I$, and let $\psi$ be the representation $\sum_{n=1}^{\infty} \oplus \psi_{n}$ of $\mathfrak{A}$ on $\mathfrak{H C}\left(=\sum_{n=1}^{\infty} \oplus \mathcal{H}_{n} \otimes \mathcal{H}_{n}\right)$. Since each $\mathfrak{H}_{n}$ is separable, $\mathcal{H C}$ is separable. Let $\phi$ be a representation of $\mathfrak{A}, a$ an abelian $C^{*}$-subalgebra of $\phi(\mathfrak{A}), \tau$ and $\eta$ pure states of $\phi(\mathfrak{A})$ whose restrictions to $Q$ are distinct pure states of $Q$, and $A$ a selfadjoint operator in $\mathfrak{A}$ with $\phi(A)$ in $a$ such that $\tau[\phi(A)] \neq \eta[\phi(A)]$. Since the irreducible representations of $\mathfrak{A}$ form a separating family [24], $\psi$ is an isomorphism; and $\tau \circ \phi \circ \psi^{-1}, \eta \circ \phi \circ \psi^{-1}$ are pure states of $\psi(\mathfrak{U})$ corresponding to distinct spectral values of $\psi(A)$. Now each pure state of $\psi(\mathfrak{A})$ gives rise to an irreducible representation of $\mathfrak{A}$ which is unitarily equivalent to some subrepresentation of $\psi$, by construction of $\psi$. Thus each pure state of $\psi(\mathfrak{A})$ corresponds to a vector in $\mathcal{H}$. In particular $\tau \circ \phi \circ \psi^{-1}, \eta \circ \phi \circ \psi^{-1}$ arise from eigenvectors for $\psi(A)$ corresponding to the distinct eigenvalues $\tau[\phi(A)], \eta[\phi(A)]$. By separability of $\mathfrak{H C}$, there are at most a countable number of pure states of a. Rosenberg $[23, \S 5]$ notes now that the pure state space of $a$ has isolated points (from Category). Since $a$ is isomorphic to the algebra of continuous functions on the pure state space $[27 ; 28]$, the function which is 1 at an isolated point and 0 elsewhere corresponds to a minimal projection $E$ in $a$. With $B$ in $a, E B E=a E$, for some scalar $a$; from the isomorphism with the function algebra. If $a$ is maximal abelian in $\phi(\mathfrak{A})$ and $T$ is a self-adjoint operator in $\phi(\mathfrak{U})$, then $E T E=b E$ or the $\mathrm{C}^{*}$-algebra generated by $E T E$ and $E$ (hence, in $\phi(\mathfrak{I})$ ) contains a smaller nonzero projection than $E$. Since $E Q E$ consists of scalar multiples of $E$, such a projection commutes with $a$ and, by maximality, lies in $a$. This would contradict the minimality of $E$ in $Q$. Thus $E \phi(\mathfrak{A}) E$ consists of scalar multiples of $E$. Since the family of such multiples is strongly closed, the mapping $T \rightarrow E T E$ is strongly continuous on the unit ball, and the unit ball in $\phi(\mathfrak{A})$ is strongly dense in the unit ball of $\phi(\mathfrak{H})^{-}[13] ; E \phi(\mathfrak{H})-E$ consists of scalar multiples of $E$. It follows that $E$ is a minimal projection in the von Neumann algebra $\phi(\mathfrak{A})^{-}$. As before, restriction to the central carrier of $E$ yields a factor of type I. Thus $\phi(\mathfrak{A})^{\prime}$ contains a minimal projection, for each representation $\phi$ of $\mathfrak{A}$, and, as before, $\mathfrak{A}$ is a type I C*-algebra.

If $\phi$ above is cyclic and $P$ is the central carrier of $E$, then $P$ is the sum of at most $\operatorname{dim}\left\{E_{1}^{\prime}(\mathcal{H C})\right\}$ pairwise orthogonal minimal projections $E_{n}^{\prime}$ in $\phi(\mathfrak{A})^{\prime}$, each with central carrier $P$. Now, the restriction of $\phi$ to $E_{1}^{\prime}$ is an irreducible representation of $\mathfrak{A}$; so, unitarily equivalent to some $\phi_{n}$. The restriction of $\phi$ to $P$ is a multiple of the restriction of $\phi$ to $E_{1}^{\prime}$ with no more copies than $\operatorname{dim}\left\{E_{1}^{\prime}(\mathcal{H C})\right\}=\operatorname{dim} \mathcal{F}_{n}$. Thus, the restriction of $\phi$ to $P$ is unitarily equivalent to a subrepresentation of $\psi_{n}$. Now $\phi$ is the direct sum of such restrictions (each pair disjoint). Thus $\phi$ is unitarily equivalent to a subrepresentation of $\psi$. From Theorem $\mathrm{C}$, the state $\omega_{x} \circ \psi$, where $x$ is a cyclic vector for $\psi(\mathfrak{A})$, is a maximal state of $\mathfrak{A}$. That $\psi$ is a cyclic representation follows 
from the fact that each $\psi_{n}$ is cyclic, that the $\psi_{n}$ are pairwise disjoint (cf. [7, Lemma 3]) and that there are at most a countable number of $\psi_{n}$. Each $\psi_{n}$ is cyclic as a result of the strong density of $\phi_{n}(\mathfrak{R})$ in all bounded operators on $\mathfrak{H}_{n}$, the separability of $\mathfrak{H C}_{n}$, and the fact that $\psi_{n}$ is a multiple of $\phi_{n}$ with dim $\mathfrak{H}_{n}$ copies. This establishes (b) $\rightarrow(a)$.

Under the hypothesis of uniform separability on $\mathfrak{A}$, the equivalence of type I with GCR is due to Glimm [6, Theorem 1]. In particular, (c) $\rightarrow$ (d). From [4], each primitive ideal in the GCR algebra $\mathfrak{A}$ corresponds to a given unitary equivalence class of irreducible representations. Thus (d) $\rightarrow$ (b); and the proof is complete.

The preceding theorem does not apply to von Neumann algebras, even those on separable Hilbert space, since they are not (in general) separable in the norm topology. On the other hand, it is not hard to see that such algebras have a maximal state if and only if they have finite dimension as vector spaces. In fact, each maximal abelian subalgebra contains a countable number of orthogonal (nonzero) projections unless it has finite dimension. The von Neumann algebra generated by these projections is isomorphic to the $\beta$-compactification of the integers [26]; and, so, has $2^{2^{c}}$ distinct pure states. Each such has a pure state extension to the von Neumann algebra. The Kaplansky argument (given in [12, p. 399]) applies now to show that there are at least an uncountable number of inequivalent irreducible unitary representations of the von Neumann algebra. However, (a) and (b) of the preceding theorem show that it cannot have a maximal state in this case. Thus each maximal abelian subalgebra has finite linear dimension. In particular, the center has, so that the identity is the sum of orthogonal minimal projections in the center. The restriction of the von Neumann algebra to each such projection is a factor. The factors whose maximal abelian algebras have finite linear dimension are, of course, just those isomorphic to a finite matrix algebra. Thus the only von Neumann algebras (on separable Hilbert space) which have maximal states are those of finite linear dimension.

We conclude with a brief outline of an extension of this theory to the case of arbitrary cyclic representations (the global theory).

Definition 4.2. If $\mathfrak{A}$ is a $C^{*}$-algebra and $\rho$ is a state of $\mathfrak{A}$ then two states $\eta$ and $\tau$ of $\mathscr{Q}$ in $\tilde{\rho}$ are said to have the same central carrier in $\tilde{\rho}$ when $\eta$ is disjoint from a state $\nu$ in $\tilde{\rho}$ if and only if $\tau$ is disjoint from $\nu$. The state $\tau$ in $\tilde{\rho}$ is central in $\tilde{\rho}$ when $\tau$ is maximal with respect to $\lesssim$ in the class of states with the same central carrier.

Remarks (4b). The states $\eta$ and $\tau$ have the same central carrier in $\tilde{\rho}$ if and only if the cyclic projections corresponding to $\eta$ and $\tau$ in $\phi_{\rho}(\mathfrak{H})^{\prime}$ all have the same central carrier.

(4c). The state $\tau$ is central in $\tilde{\rho}$ if and only if the class of cyclic projections corresponding to $\tau$ in $\phi_{\rho}(\mathfrak{U})^{\prime}$ contains a central projection.

The difficulty we encountered in distinguishing those states which give 
factor representations of type III and those which give one-dimensional representations has its global analogue. In this case, states $\rho$ such that $\phi_{\rho}(\mathfrak{A})$ is abelian (i.e., of type $I_{1}$ ) take the place of the homomorphisms. Such states are described in terms of the state space in the following:

Definition 4.3. A state $\rho$ of a $C^{*}$-algebra $\mathfrak{A}$ is said to be of type $\mathrm{I}_{1}$ when $\tilde{\tau}=\bar{\tau}$ for each $\tau$ in $\tilde{\rho}$. The state $\tau$ in $\tilde{\rho}$ is said to be a type III component of $\tilde{\rho}$ when $\tau$ is not of type $\mathrm{I}_{1}$ and is both maximal and minimal relative to $\lesssim$ in the class of states with the same central carrier. The state $\tau$ in $\tilde{\rho}$ is said to be abelian in $\tilde{\rho}$ when it is not of type III in $\tilde{\rho}$ and is minimal relative to $\lesssim$ in the class of states with the same central carrier. The state $\tau$ in $\tilde{\rho}$ is said to be a type I component of $\tilde{\rho}$ when $\tau$ is central in $\tilde{\rho}$ and has the same central carrier as some abelian state in $\tilde{\rho}$. The state $\tau$ in $\tilde{\rho}$ is said to be a type II component of $\tilde{\rho}$ when $\tau$ is central in $\tilde{\rho}$ and contains no type I or III components of $\tilde{\rho}$.

REMARKs (4d). A state $\tau$ in $\tilde{\rho}$ is a component of $\tilde{\rho}$ of type I, II, or III, if and only if the class of cyclic projections in $\phi_{\rho}(\mathfrak{A})^{\prime}$ corresponding to $\tau$ contains a central projection $P$ such that $\phi_{\rho}(\mathfrak{A})^{\prime} P$ is of type I, II, or III, [15] respectively.

(4e). The state $\tau$ in $\tilde{\rho}$ is abelian in $\tilde{\rho}$ if and only if each of the cyclic projections corresponding to $\tau$ in $\phi_{\rho}(\mathfrak{H})^{\prime}$ is abelian [15] in $\phi_{\rho}(\mathfrak{A})^{\prime}$.

(4f). Standard techniques allow us to locate three states in $\tilde{\rho}$ uniquely described by the property of being the maximal components of $\tilde{\rho}$ of types $I$, II, and III, respectively. Moreover, no state in $\tilde{\rho}$ is disjoint from all three. These states are called the components of types I, II, and III, respectively.

\section{BIBLIOGRAPHY} 1957.

1. J. Dixmier, Les algèbres d'opérateurs dans l'espace Hilbertien, Gauthier-Villars, Paris,

2. - Les anneaux d'opérateurs de classe fini, Ann. École Norm. (3) 66 (1949), 209-261.

3. H. Dye, The Radon-Nikodym theorem for finite rings of operators, Trans. Amer. Math. Soc. 72 (1952), 243-280.

4. J. Fell, $C^{*}$-algebras with smooth dual, Illinois J. Math. 4 (1960), 221-230.

5. I. Gelfand and $M$. Neumark, On the imbedding of normed rings into the ring of operators on Hilbert space, Rec. Math. (Mat. Sb.) N.S. 12 (1943), 197-213.

6. J. Glimm, Type I $C^{*}$-algebras, Ann. of Math. 73 (1961), 572-612.

7. J. Glimm and R. Kadison, Unitary operators in $C^{*}$-algebras, Pacific J. Math. 10 (1960), 547-556.

8. E. Griffin, Some contributions to the theory of rings of operators, Trans. Amer. Math. Soc. 75 (1953), 471-504.

9. - Some contributions to the theory of rings of operators. II, Trans. Amer. Math. Soc. 79 (1955), 389-400.

10. R. Kadison, Unitary invariants for representations of operator algebras, Ann. of Math. 66 (1957), 304-379.

11. - Irreducible operator algebras, Proc. Nat. Acad. Sci. U.S.A. 43 (1957), 273-276.

12. R. Kadison and I. Singer, Extensions of pure states, Amer. J. Math. 81 (1959), 383-400.

13. I. Kaplansky, $A$ theorem on rings of operators, Pacific J. Math. 1 (1951), 227-232.

14. - The structure of certain operator algebras, Trans. Amer. Math. Soc. 70 (1951), 219-255. 
15. - Projections in Banach algebras, Ann. of Math. 53 (1951), 235-249.

16. G. Mackey, Induced representations of locally compact groups. II. The Frobenius reciprocity theorem, Ann. of Math. 58 (1953), 193-221.

17. F. Mautner, Unitary representations of locally compact groups. II, Ann. of Math. 52 (1950), 528-556.

18. F. Murray and J. von Neumann, On rings of operators, Ann. of Math. 37 (1936), 116229.

19. - On rings of operators. II, Trans. Amer. Math. Soc. 41 (1937), 208-248.

20. J. von Neumann, Zur Algebra der Funktionaloperatoren und Theorie der normalen Operatoren, Math. Ann. 102 (1930), 370-427.

21. - On rings of operators. Reduction theory, Ann. of Math. 50 (1949), 401-485.

22. L. Pukánszky, On a theorem of Mautner, Acta Sci. Math. Szeged 15 (1954), 145-148.

23. A. Rosenberg, The number of irreducible representations of simple rings with no minimal ideals, Amer. J. Math. 75 (1953), 523-530.

24. I. Segal, Irreducible representations of operator algebras, Bull. Amer. Math. Soc. 53 (1947), 73-88.

25. - Two-sided ideals in operator algebras, Ann. of Math. 50 (1949), 856-865.

26. M. Stone, Applications of the theory of Boolean rings to general topology, Trans. Amer. Math. Soc. 41 (1937), 375-481.

27. — A general theory of spectra. I, Proc. Nat. Acad. Sci. U.S.A. 26 (1940), 280-283.

28. - A general theory of spectra. II, Proc. Nat. Acad. Sci. U.S.A. 27 (1941), 83-87.

Columbia University,

New York, New York 